\title{
Prefrontal Microcircuits: Membrane Properties and Excitatory Input of Local, Medium, and Wide Arbor Interneurons
}

\author{
Leonid S. Krimer and Patricia S. Goldman-Rakic \\ Section of Neurobiology, Yale University School of Medicine, New Haven, Connecticut 06520-8001
}

To elucidate cortical mechanisms involved in higher cortical functions such as working memory, we have examined feedforward excitation transmitted by identified pyramidal cells to interneurons with predominantly horizontal axonal arbors, using dual somatic recordings in prefrontal cortical slices. Interneurons with local (narrow) axonal arbors, especially chandelier interneurons, exhibited extremely narrow action potentials and high evoked firing rates, whereas neurons identified with wide arbor axons generated wider spikes and lower evoked firing rates with considerable spike adaptation, resembling that of pyramidal cells. Full reconstruction of differentially labeled neuronal pairs revealed that local arbor cells generally received a single but functionally reliable putative synaptic input from the identified pyramidal neuron member of the pair. In contrast, more synapses (two to five) were necessary to depolarize medium and wide arbor neurons reliably. The number of putative synapses and the amplitude of the postsynaptic response were remarkably highly correlated within each class of local, medium, and wide arbor interneurons $(r=0.88,0.95$, and 0.99, respectively). Similarly strong correlations within these subgroups were also present between the number of putative synapses and variance in the EPSP amplitudes, supporting the validity of our morphological analysis. We conclude that interneurons varying in the span of their axonal arbors and hence in the potential regulation of different numbers of cortical modules differ also in their excitatory synaptic input and physiological properties. These findings provide insight into the circuit basis of lateral inhibition and functional interactions within and between cortical columns in the cerebral cortex.

Key words: interneurons; membrane properties; unitary EPSPs; synaptic efficacy; paired recordings; synaptic number; prefrontal cortex
Local circuit neurons of the cerebral cortex participate in structuring receptive fields of pyramidal cells via inhibitory GABAergic neurotransmission. In visual cortex they contribute to orientation and direction tuning of excitatory neurons (Sillito, 1975; Eysel et al., 1998). Lateral inhibition, including oblique- and cross-orientation inhibition (Kisvarday et al., 1994), has been hypothesized to account for these effects (Sillito, 1984; Eysel, 1992). Recent evidence from this laboratory indicates that similar inhibitory mechanisms operate in the prefrontal cortex to shape the memory fields of neurons that are engaged in working memory tasks (Wilson et al., 1994; Rao et al., 1999, 2000).

Another point of interest is the recent evidence that pyramidal neurons project preferentially onto nearby, but not onto distant (>250 $\mu \mathrm{m}$ ), interneurons (Elhanany and White, 1990; McGuire et al., 1991; Melchitzky et al., 1998; Muly et al., 1998). As such, it appears that a major mode, perhaps the major mode of intercolumnar inhibition of both pyramidal and nonpyramidal neurons, is via local pyramidal-interneuronal circuits. Supporting this organization, GABAergic fast-spiking interneurons recently have been found to discharge only in synchrony with neighboring neurons, indicating that local excitatory input to interneurons may be the only input driving the inhibitory network in the

Received Jan. 19, 2001; revised March 13, 2001; accepted March 14, 2001.

This work was supported by National Institutes of Health Grants MH 38546, MH 44866, and MH 63561-01. We express appreciation to Dr. Graham Williams and Dr. Ethan D. Cohen for valuable technical advice on the physiological set-up in early stages of this research, to Dr. David McCormick for a generous donation of ferret tissue that was used in this study, and to Dr. James Howe for valuable comments on this manuscript.

Correspondence should be addressed to Patricia S. Goldman-Rakic, Section of Neurobiology, Yale University School of Medicine, P.O. Box 208001, New Haven, CT 065120-8001. E-mail: patricia.goldman-rakic@yale.edu.

Copyright @ $\odot 2001$ Society for Neuroscience $0270-6474 / 01 / 213788-09 \$ 15.00 / 0$ regulation of rhythmic excitation as well as constraining epileptiform activity (Sanchez-Vives and McCormick, 2000).

Given the signal importance of inhibition in cortical function, the present study was designed to examine further the intrinsic circuits in prefrontal cortex, with a focus on the interactions between principal neurons and local circuit neurons in this cortex, which single-unit recording, lesion, and imaging studies have shown to subserve mnemonic functions.

Such interactions have been characterized recently in other cortical areas, using a paired recording technique (Thomson et al., 1995; Buhl et al., 1997; Galarreta and Hestrin, 1998; TarczyHornoch et al., 1998; Angulo et al., 1999), and distinct physiological properties of excitatory synapses have been associated with different types of postsynaptic neuron (Reyes et al., 1998). We here examine, in prefrontal cortex, the local excitatory input to three groups of interneurons with predominantly horizontal axonal arbors: local arbor cells (LACs), medium arbor cells (MACs), and wide arbor cells (WACs), similar to those described in primate prefrontal cortex by Lund and Lewis (1993) and in rat by Kawaguchi (1995). Such neurons represent a large population of interneurons in the prefrontal cortex and are likely to play distinct functional roles because of the differential range of influence conveyed by the length of their axonal arbors. Although LACs (including chandelier interneurons) with narrow, $\sim 300 \mu \mathrm{m}$, axonal arbors may provide inhibitory effects within narrow cortical modules, medium (500 $\mu \mathrm{m}$ axonal extent) and especially wide arbor (>1000 $\mu \mathrm{m}$ axonal extent) neurons could influence neighboring modules (Bugbee and Goldman-Rakic, 1983; GoldmanRakic, 1984; Lund and Lewis, 1993; Melchitzky et al., 2001).

Our findings indicate that interneurons differentiated by the span of their horizontal axonal arbors could be distinguished 
further on the basis of their physiological membrane properties as well as on the properties of their local excitatory input. This study also examined the structure-function relationships between physiological response and excitatory input by full anatomical reconstruction of virtually every physiologically characterized pair of neurons.

\section{MATERIALS AND METHODS}

Preparation. Twenty connected neuronal pairs were identified in slices of medial prefrontal cortex taken from 1.5- to 4-month-old ferrets. Two of the pairs were excluded from further analysis because we were unable to recover in full the axons of their presynaptic pyramids in our anatomical reconstructions. However, two additional small arbor neurons and two chandelier cells (ChCs) that were recorded individually were included in the analysis of morphological and membrane properties.

The animals were anesthetized deeply with sodium pentobarbital and decapitated. The brain was removed quickly and chilled for a few minutes in ice-cold Ringer's solution. The frontal lobe was separated, and $400 \mu \mathrm{m}$ sagittal sections were cut through the medial prefrontal region on a DTK-1500E microslicer (Dosaka, Kyoto, Japan). Sections were incubated at $35^{\circ} \mathrm{C}$ and after at least $1.5 \mathrm{hr}$ were submerged in the perfusion chamber at $31-32^{\circ} \mathrm{C}$. Extracellular solution contained (in $\mathrm{mM}$ ): 126 $\mathrm{NaCl}, 2.5 \mathrm{KCl}, 1.25 \mathrm{NaH}_{2} \mathrm{PO}_{4}, 2 \mathrm{CaCl}_{2}, 1 \mathrm{MgSO}_{4}, 26 \mathrm{NaHCO}_{3}$, and 10 dextrose, $\mathrm{pH} 7.4$, perfused with a $95 \% \mathrm{O}_{2} / 5 \% \mathrm{CO}_{2}$ gas mixture. Structures were visualized by using infrared differential interference contrast video microscopy as described previously (Stuart et al., 1993). Interneurons of laminae II/III were selected visually on the basis of their lack of an apical dendrite and small soma size (relative to pyramidal cells).

Physiological analysis. Dual whole-cell voltage recordings were used for analysis of pyramidal-to-nonpyramidal monosynaptic connections. Patch electrodes with electrical resistances of $10-12 \mathrm{M} \Omega$ were filled with a solution containing (in $\mathrm{mm}$ ): $114 \mathrm{~K}$-gluconate, $6 \mathrm{KCl}, 0.5 \mathrm{CaCl}, 1$ EGTA, 4 ATP-Mg, and $10 \mathrm{HEPES}$, pH-adjusted to 7.25 with $\mathrm{KOH}$. Lucifer yellow ( $0.2 \%$, dipotassium salt; Sigma, St. Louis, MO) was added for morphological identification of interneurons, and $0.5 \%$ biocytin (Molecular Probes, Eugene, OR) was used to label pyramidal cells. Membrane was broken by suction at $\geq 5 \mathrm{G} \Omega$ seal resistance. Both access resistance and capacitance were compensated on-line. Access resistance was determined from the settings of the bridge balance from the currentclamp amplifiers (Markram et al., 1997) and typically was 15-25 M $\Omega$. It was stable during recordings, as indicated by low noise $(0.11 \pm 0.02 \mathrm{mV})$ and its small variance $(\mathrm{CV}=18 \%)$. The root mean square $(\mathrm{rms})$ noise was measured for 15 neuronal pairs from the baseline within $30 \mathrm{msec}$ before EPSP onset. Sweeps contaminated with spontaneous EPSPs were eliminated for all measurements. Pyramidal cells within $\sim 100 \mu \mathrm{m}$ were tested for monosynaptic connections to a given interneuron. They were stimulated with narrow 10 -msec-duration rectangular suprathreshold current pulses (Markram et al., 1997) at $1 \mathrm{~Hz} ; 20$ trials were averaged on-line to confirm connectivity. Then 20-50 single trials were recorded at $0.17-0.25 \mathrm{~Hz}$ for further off-line analysis. EPSPs were measured as follows: latencies, from peak of presynaptic spike to the onset of EPSPs; amplitudes, from baseline to peak of EPSPs; rise time, from onset of EPSPs to its peak; and $50 \%$ decay, from the peak of EPSPs to its half value. Time constant was calculated from a curve fitting to the repolarization slope of EPSPs and best described as first-order exponential decay. There was no EPSP run-down over the tested time range of 8-20 min $(n=$ 4). The method of whole-cell recording that was used allowed for low noise data acquisition with reliable detection of evoked postsynaptic events. There was almost no overlap between noise and EPSP amplitudes for all of our recordings (Fig. 1), and that enabled accurate detection of the failure incidence among the identified synaptic transmissions. Accordingly, there was no correlation between the percentage of failures and the magnitude of rms noise (coefficient of correlation, 0.08).

To characterize electrical membrane properties of neurons, we applied hyper-and depolarizing rectangular current pulses of $500 \mathrm{msec}$ duration in $50 \mathrm{pA}$ increments (Kawaguchi, 1995). Single action potentials also were evoked by single 30 -msec-duration depolarizing current pulses. In general, postsynaptic nonpyramidal neurons satisfied the physiological criteria of interneurons (McCormick et al., 1985, Chen et al., 1996). They typically had narrow spikes $(0.43 \pm 0.07 \mathrm{msec})$ with amplitudes, on average, of $25 \mathrm{mV}$ smaller ( $80 \pm 7 \mathrm{mV}$ as measured from the baseline) than those of pyramidal cells. Interneurons discharged at $2-36 \mathrm{~Hz}$ in response to just-above-threshold stimulation, with the first spike consistently occurring at the beginning of the current pulse. Stronger stimula-

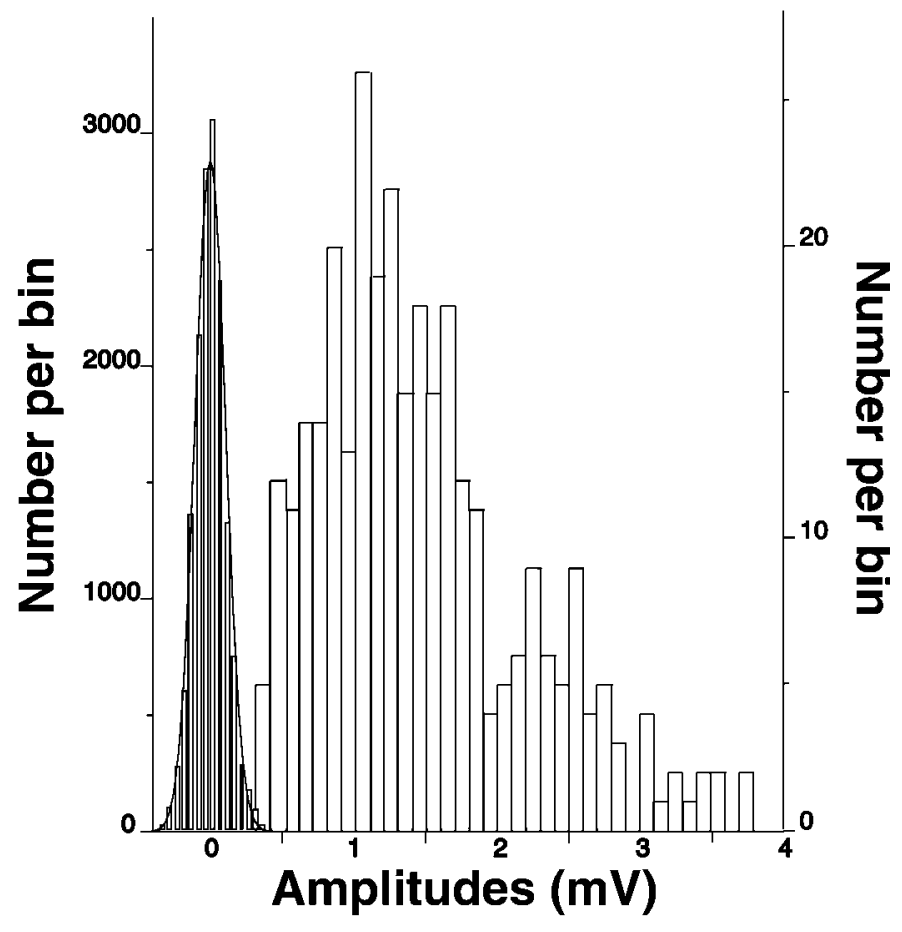

Figure 1. Distribution of amplitudes for noise (on the left) and for EPSPs recorded in postsynaptic interneurons. There is almost no overlap in amplitude distributions between noise and EPSPs for all of the recorded pairs.

tion currents further demonstrated the high firing rates of these cells and the lack of significant spike adaptation for most of these cells. Interneurons had membrane potentials of $-67 \pm 4 \mathrm{mV}$ (measurements were not compensated for liquid junction potential), input resistances of $109 \pm 29$ $\mathrm{m} \Omega$, and time constants of $10.5 \pm 2.8 \mathrm{msec}$ (the last two parameters were measured on sweeps produced by $50 \mathrm{pA}$ hyperpolarizing pulses). For pyramidal cells the average values were membrane potential, $-69 \pm 5$ $\mathrm{mV}$; input resistance, $101 \pm 45 \mathrm{~m} \Omega$; time constant, $26.2 \pm 12.2 \mathrm{msec}$; action potential amplitude, $105 \pm 9 \mathrm{mV}$; and action potential width, $1.11 \pm 0.18 \mathrm{msec}$. All pyramidal cells generated considerably lower firing frequencies (than interneurons), with prominent spike adaptation in response to 500-msec-depolarizing injected currents.

Voltages were amplified and filtered at $2 \mathrm{kHz}$, using two Intracellular Electrometers IE-210 (Warner Instrument, Hamden, CT) operating in bridge balance mode, and acquired on the computer at sampling rates of $10-30 \mathrm{kHz}$, using DigiData 1200 interface and pClamp 6 software program (Axon Instruments, Foster City, CA). In addition to pClamp 6, Axoscope (Axon Instruments), Microsoft Excel, and Origin 5.0 software programs were used for data analysis. The data are presented in average \pm SD values throughout the text. For statistical analysis two-sample Student's $t$ test was used unless otherwise specified.

Morphological analysis. After recordings, the pairs were left patched for a total of 1-2 hr for good dye labeling of their dendritic and axonal arbors. Free-floating slices were fixed in cold $4 \%$ paraformaldehyde for $72 \mathrm{hr}$, transferred into anti-freezing solution (mixture of ethylene glycol and glycerol in $0.1 \mathrm{M}$ phosphate buffer), and stored at $-79^{\circ} \mathrm{C}$. Later they were sectioned into $5 \times 60 \mu \mathrm{m}$ sections on the microslicer. Sections were reacted with $1 \% \mathrm{H}_{2} \mathrm{O}_{2}$ and placed in blocking serum with $0.5 \%$ Triton $\mathrm{X}-100$ at $4^{\circ} \mathrm{C}$ for $12 \mathrm{hr}$. Then they were incubated in avidin-biotin complex $(\mathrm{ABC})$ for $4 \mathrm{hr}$ at room temperature and, after being rinsed, were transferred to a solution of anti-Lucifer yellow biotinylated rabbit IgG (Molecular Probes) for $48 \mathrm{hr}$. Biocytin-labeled pyramids were developed by using the black Ni-DAB chromogen. Lucifer yellow-labeled interneurons were stained golden brown, using a plain diaminobenzidine (DAB) reaction after additional $\mathrm{ABC}$ incubation. To avoid extensive shrinkage in thickness, we dehydrated sections while they were free-floating.

Neuronal three-dimensional reconstruction and morphometric measurements of dendritic and axonal arbors were made with Neurolucida 
Figure 2. Morphological analysis of recorded neuronal pairs. $a$, Low-power photomicrograph. The presynaptic pyramidal neuron (black) was loaded with biocytin and reacted with NI-DAB; the postsynaptic medium arbor basket neuron (black) was filled with Lucifer yellow and developed with DAB. $b$, Highpower microphotographs depicting en passe and terminal putative synapses established by axons (black) of the presynaptic pyramids on the dendrites (brown) of the postsynaptic interneurons. $c, d$, Examples of three-dimensional reconstructed neuronal pairs of presynaptic pyramids and postsynaptic interneurons from the group of small interneurons. $c$, Small arbor basket cell. $d$, Small chandelier cell. Note that presynaptic pyramids (dendrites are orange, and axons are green) establish only one putative synapse (white circle) on these small postsynaptic interneurons (dendrites are light blue, and axons are darker blue).
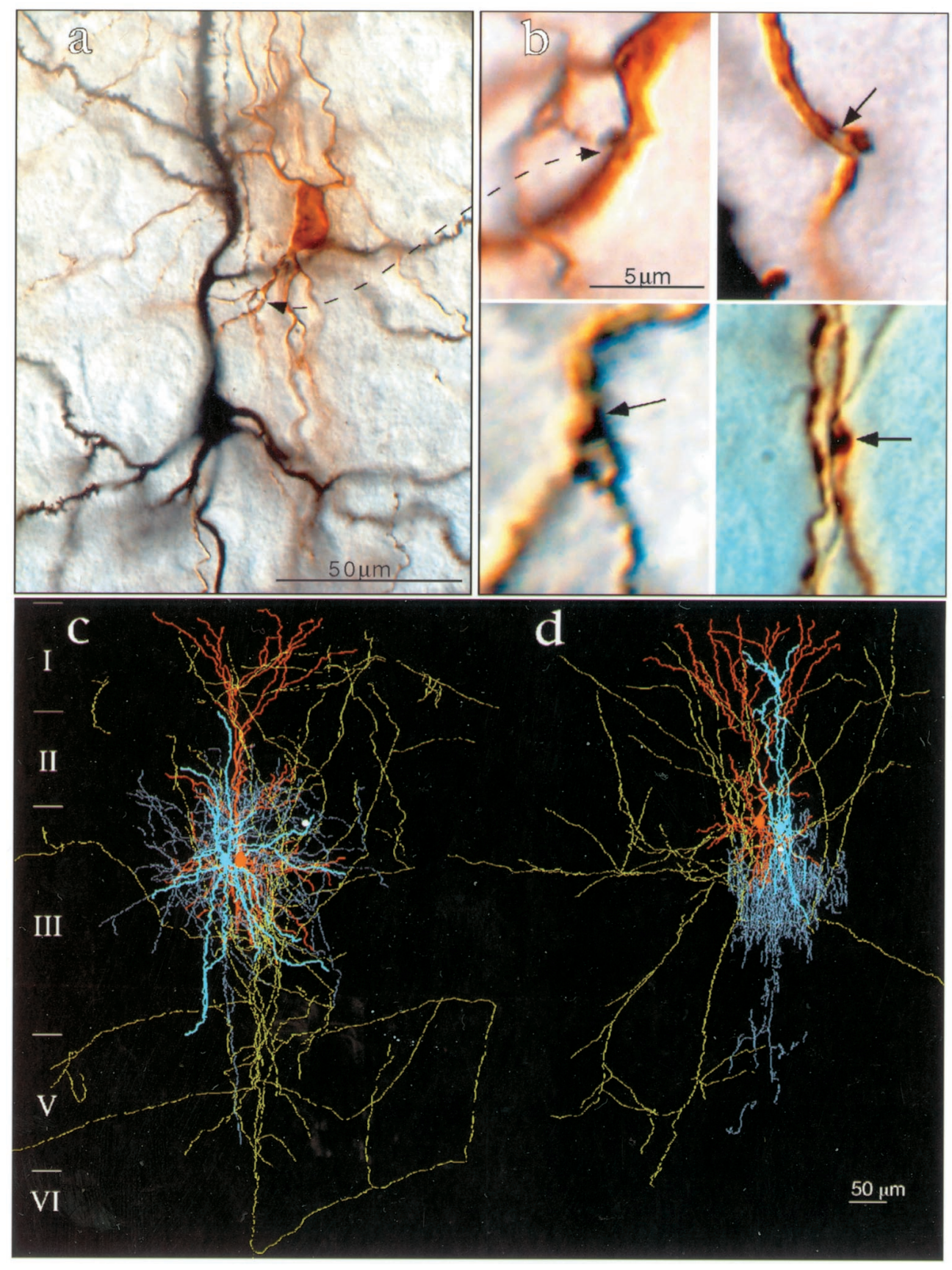

software (MicroBrightField, Colchester, VT) and an optical set-up as described previously (Krimer et al., 1997). To avoid significant deterioration of optical resolution deep in the sections, we mounted them between two coverslips and analyzed them on both sides. The double immunolabeling procedure that was used allowed for clear color distinction between pyramidal and nonpyramidal neurons, reliable cell reconstruction, and analysis of putative synaptic contacts (Fig. 2a,b). In fact, the color differentiation was so clear (especially when focusing up and down along the axons and observing the color of their boutons) that we easily could distinguish putative synapses (black) from multiple autapses on the interneurons (stained brown). In addition, as a conservative measure, we also traced axons with putative contacts on interneurons back to their pyramidal cell origins to further reassure the accuracy of the analysis. Morphometric analysis for recorded neurons included measurements of soma perimeter, total dendritic and axonal length, and distance from soma to the pia and to the soma of the other connected neuron. To classify interneurons accurately, we further characterized their axonal arbors for width, height, area, and density of distribution. Pyramidal neurons had distinctive morphological features, namely the presence of apical and basal dendrites with numerous spines. However, the morphology of their dendritic and local axonal arbors exhibited substantial variation (see Figs. 2, 3). The quantitative measurements were not corrected for tissue shrinkage, which is known to be $\sim 30 \%$ for free-floating dehydration of cortical slices (Krimer et al., 1997).

\section{RESULTS}

\section{Anatomy of interneurons}

The three-dimensional reconstructions and morphometric measurements revealed three classes of interneurons that are based on the size of their soma and width of axonal arbor cells with narrow or local axonal arbors (LACs), medium arbor cells (MACs), and wide arbor cells (WACs), a classification very similar to that reported in a previous Golgi analysis of layer III pyramidal neurons in the primate prefrontal cortex (Lund and Lewis, 1993). The soma of LACs were small, and their horizontal 


\begin{tabular}{|c|c|c|c|c|c|c|c|c|c|}
\hline $\begin{array}{l}\text { Groups of } \\
\text { interneurons }\end{array}$ & $\begin{array}{l}\text { Types of } \\
\text { interneurons }\end{array}$ & $\begin{array}{l}\text { Laminar } \\
\text { position }\end{array}$ & $\begin{array}{l}\text { Distance to } \\
\text { pia }(\mu \mathrm{m})\end{array}$ & $\begin{array}{l}\text { Distance to } \\
\text { pyramid } \\
(\mu \mathrm{m})\end{array}$ & $\begin{array}{l}\text { Width of axonal } \\
\text { arbor }(\mu \mathrm{m})\end{array}$ & $\begin{array}{l}\text { Soma perimeter } \\
(\mu \mathrm{m})\end{array}$ & $\begin{array}{l}\text { Number of } \\
\text { putative } \\
\text { synapses }\end{array}$ & $\begin{array}{l}\text { EPSP } \\
\text { amplitudes } \\
(\mathrm{mV})\end{array}$ & $\begin{array}{l}\text { Failures } \\
(\%)\end{array}$ \\
\hline \multirow[t]{9}{*}{ LACs } & LAC & IIIs & 360 & 35 & 340 & 39 & 1 & 0.5 & $-^{a}$ \\
\hline & LAC & IIIs & 283 & 44 & 364 & 39 & 1 & $0.6 \pm 0.25$ & 20 \\
\hline & LAC & III & 445 & 33 & 400 & 42 & 1 & $0.8 \pm 0.29$ & 7 \\
\hline & LAC & II & 222 & 63 & 312 & 35 & 1 & $1.0 \pm 0.37$ & 0 \\
\hline & LAC & IIIs & 343 & 22 & 300 & 40.3 & 1 & $1.0 \pm 0.30$ & 15 \\
\hline & $\mathrm{ChC}$ & IIIs & 299 & 29 & 163 & 40.2 & 1 & $1.0 \pm 0.47$ & 50 \\
\hline & LAC & II & 252 & 37 & 314 & 38 & 1 & $1.1 \pm 0.29$ & 26 \\
\hline & LAC & III & 397 & 49 & 372 & 36 & 2 & $1.9 \pm 0.73$ & 12 \\
\hline & LAC & IIIs & 309 & 36 & 320 & 39 & 3 & $2.0 \pm 0.75$ & 0 \\
\hline \multirow[t]{7}{*}{ MACs } & MAC & II & 185 & 32 & 845 & 48 & 2 & $1.5 \pm 0.6$ & 6 \\
\hline & MAC & IIIs & 302 & 51 & 544 & 46 & 2 & $1.5 \pm 0.72$ & 18 \\
\hline & MAC & II & 234 & 15 & 539 & 46.1 & 3 & $1.7 \pm 0.52$ & 12 \\
\hline & MAC & IIIs & 350 & 64 & 470 & 50.1 & 4 & $2.1 \pm 0.55$ & 0 \\
\hline & MAC & II & 251 & 58 & 530 & 46.2 & 5 & $2.5 \pm 0.78$ & 0 \\
\hline & MAC & II & 256 & 23 & 530 & 44 & 1 & $0.5 \pm 0.29$ & 50 \\
\hline & MAC & IIIs & 365 & 19 & 686 & 50 & - & 1.0 & $-^{a}$ \\
\hline \multirow[t]{4}{*}{ WACs } & WAC & II & 242 & 25 & - & 55 & - & $1.1 \pm 0.23$ & 0 \\
\hline & WAC & IIIs & 275 & 23 & 1078 & 61.7 & 3 & $1.0 \pm 0.35$ & 0 \\
\hline & WAC & IIIs & 266 & 29 & 852 & 59 & 5 & $1.5 \pm 0.56$ & 3 \\
\hline & WAC & II & 212 & 46 & 640 & 57.3 & 1 & $0.3 \pm 0.11$ & 42 \\
\hline
\end{tabular}

${ }^{a}$ Only on-line averaging of 20 individual sweeps was available for EPSP measurements in these two pairs; therefore, percentage of failures could not be calculated.

axonal arbors were, on average, $\sim 300 \mu \mathrm{m}$ in width (Fig. $2 c$, Table 1). Their labeled axons consistently had numerous and well developed boutons, many of which contacted unidentified cell bodies and/or proximal dendrites of pyramidal cells as visualized under differential interference contrast. Among LAC, the ChC had the narrowest horizontal axonal span with $>100$ reconstructed axonal cartridges arranged in the classical vertical manner known to target the axon initial segments of an equal number of pyramidal cells (Fig. 2d, Table 1) (Williams et al., 1992). The dendrites of LACs were usually smooth, relatively linear, and slightly biased vertically with branching points close to the soma, and that of the chandelier cell had a notably narrow vertical course. However, their high axonal density in tissue volume was relatively high $\left(0.23\right.$ and $\left.0.26 \mu \mathrm{m} / \mu \mathrm{m}^{3}\right)$, compatible with extensive influence over multiple cells in the local vicinity.

MACs and WACs had significantly larger cell bodies, and their symmetrical axonal arbors covered a wider span of cortical modules than those of the LACs, with $\sim 600$ and $900 \mu \mathrm{m}$ symmetrical horizontal axonal spans, respectively (Table 1, Fig. 3a,c). Although the area of their axonal distribution was considerably larger than for LACs, their axonal density per tissue volume was almost twofold lower ( 0.16 vs $0.26 \mu \mathrm{m} / \mu \mathrm{m}^{3}$, respectively). This anatomical finding suggests that, although MACs and WACs are likely to control wider cortical expanses, they may have weaker effects on their targets. WACs in particular are in position to influence several cortical modules. Interestingly, their axons made both local and remote arborizations (Fig. $3 c$ ). The local plexus was very similar to that of LACs in total axonal length, height, width, and density, whereas their long-range axons gave off short and rather scarce branches along their trunks.

\section{Membrane properties of interneurons}

LACs and $\mathrm{ChCs}$, in particular, were the fastest spiking interneurons under study. In line with previous reports (Kawaguchi,
1995), LACs generated narrow spikes with widths of $0.42 \pm 0.07$ msec and high firing rates of $40.4 \pm 7.47$ (Fig. 4). Evoked trains of action potentials did not show spike adaptation. The membrane properties of three chandelier cells were also distinct not only from the rest of interneurons but even from the other LACs (Fig. 4). Their firing rates were $42 \%$ higher $(p<0.01$; Mann-Whitney $U$ test) and the action potentials were narrower $[0.35 \pm 0.01 \mathrm{msec}$; $p<0.04$; Mann-Whitney $U$ test) than those of other LACs. The membrane properties of MACs were very similar to those of LACs. They displayed fast-spiking rates with action potentials and no noticeable adaptation between action potentials. However, they tended to generate slightly wider spikes $(0.47 \pm 0.035)$ with lower evoked firing rates $(33 \pm 6 \mathrm{~Hz}$; Fig. 4) than LACs. WACs had the lowest firing rates $(16 \pm 10.6 \mathrm{~Hz})$ and the widest spikes $(0.52 \pm 0.02 \mathrm{msec})$ of all nonpyramidal cell classes that were examined. They also displayed considerable spike adaptation (Fig. 4). In fact, their firing pattern was very similar to that of pyramidal cells. However, WACs still could be distinguished easily from the latter on the basis of their twofold narrower spikes. The latter were also $\sim 25 \mathrm{mV}$ smaller in amplitude than the action potentials of pyramidal cells.

\section{Local excitatory unitary input to interneurons}

Approximately every fourth pyramidal cell that was tested in laminae II/IIIa established monosynaptic connections with an interneuron within $100 \mu \mathrm{m}$ of its soma. All of the evoked EPSPs had short latencies of $1.2 \pm 0.35 \mathrm{msec}$. In general, the evoked postsynaptic response was quite strong, with the amplitude of the unitary EPSPs across all pairs averaging $1.3 \pm 0.56 \mathrm{mV} ; 15$ pairsexhibited EPSPs at or above $1 \mathrm{mV}$ (see Fig. 1, Table 1). Among the latter, eight pairs had EPSP amplitudes between 1.5 and $2.5 \mathrm{mV}$, and some of the individual postsynaptic responses reached $4 \mathrm{mV}$. Only five pairs had postsynaptic responses $<0.8$ $\mathrm{mV}$. The EPSPs had fast rise times $(1.4 \pm 0.40 \mathrm{msec})$ and short 


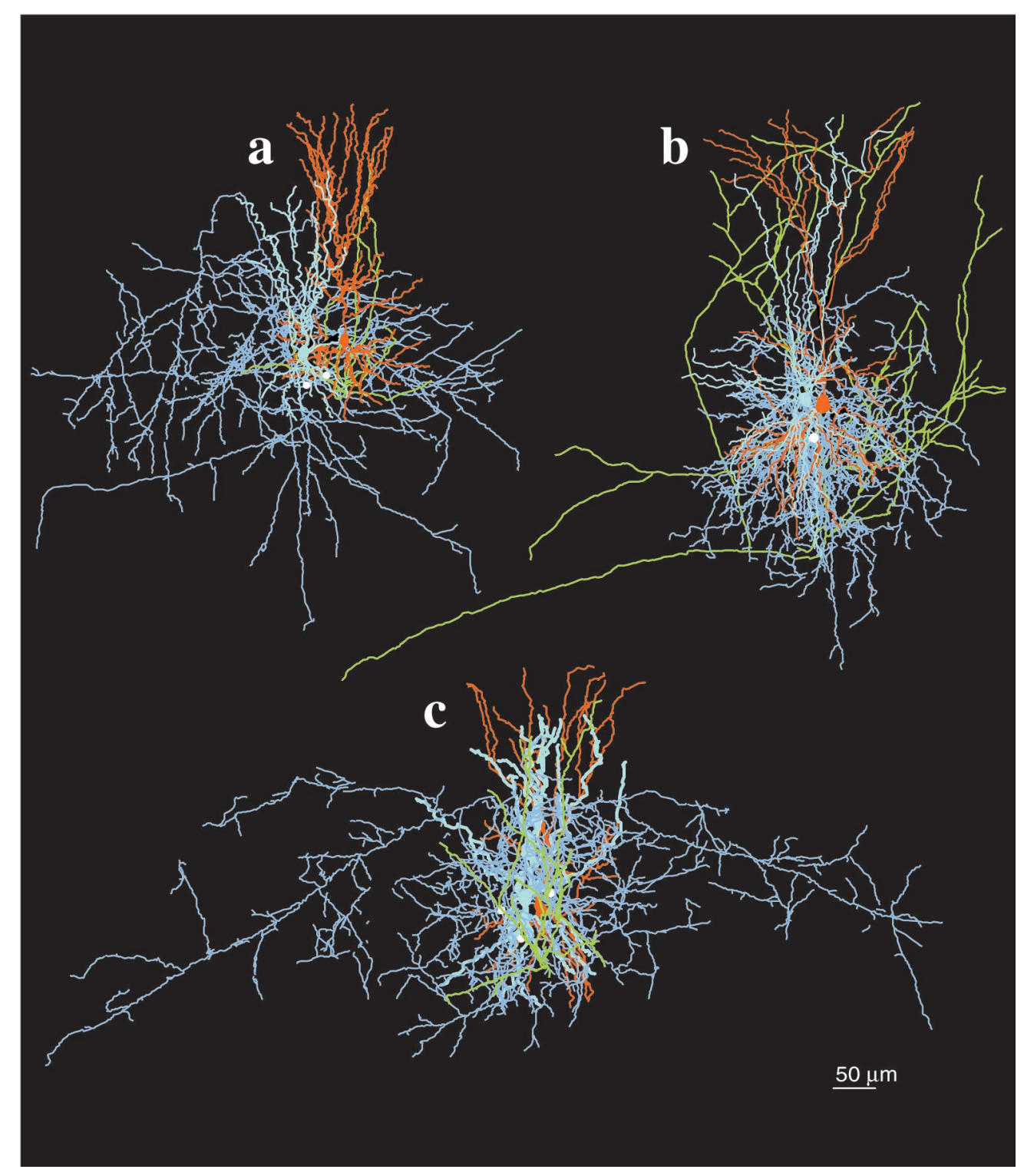

Figure 3. Three-dimensional reconstruction of monosynaptically connected neuronal pairs of presynaptic pyramids and postsynaptic interneurons from groups of medium and wide arbor interneurons. $a$, Medium arbor basket cell. $b$, Dendrite-targeting cell. $c$, Wide arbor basket cell. Note that presynaptic pyramids (dendrites are orange, and axons are yellow) establish two and three putative synapses (white circles) on medium and wide arbor basket cells, respectively (dendrites are light blue, and axons are darker blue).

duration $(50 \%$ decay time of $7.5 \pm 2.65 \mathrm{msec})$. In all pairs, the evoked EPSPs had a first-order exponential decay of $\tau=10.1 \pm$ $2.85 \mathrm{msec}$. Synaptic transmission from the pyramids to interneurons was remarkably reliable and failed, on average, in $15 \pm 19 \%$ of the trials. Only three recorded pairs demonstrated a high rate of failures, i.e., $\geq 42 \%$ (Table 1 ).

Pyramidal-to-LAC neurotransmission generally was accomplished via a single synaptic contact and was remarkably reliable. Only one putative synapse was found in seven of nine pairs of LACs (see Fig. 2, Table 1). These connections had mean amplitudes of $0.9 \mathrm{mV}$ (Fig. 5) and, with the exception of the ChC, only $11.4 \pm 9.8 \%$ of neurotransmission failures. The average variance in EPSP amplitude was relatively low $(\mathrm{SD}=0.3 \mathrm{mV})$. The remaining two LAC pairs had two and three putative synaptic connections, respectively. Remarkably, their mean EPSP amplitudes were twofold larger $(1.95 \mathrm{mV} ; p<0.0001)$ as were their average variances $(\mathrm{SD}=0.74 \mathrm{mV} ; p<0.00003)$. The $\mathrm{ChC}$ was different from all of the other LACs recorded here in having a high neurotransmission failure rate of $50 \%$.

Pyramidal-to-MAC unitary connections were multisynaptic (see Figs. 3, 5, Table 1). The number of putative synapses on
MACs varied between two and five, significantly more than on LACs $(p<0.05)$. These connections, with one exception, were functionally very reliable, with $1.9 \pm 0.6 \mathrm{mV}$ mean EPSP amplitude and $7.2 \pm 7.8 \%$ neurotransmission failures. Pyramidal-toWAC unitary connections were similar to MACs but had somewhat smaller amplitudes (see Figs. 3, 5, Table 1). Exceptions to these findings were observed in two cases in which only a single putative synapse could be identified in the reconstruction of axonal contacts. In both instances (one MAC and one WAC) we identified notably weak (mean amplitudes of $0.5 \pm 0.29$ and $0.3 \pm$ $0.11 \mathrm{mV}$, respectively) and unreliable (50 and $42 \%$, respectively) connections. We attribute the unusually low amplitude EPSPs and high failure rates in these cases to the loss of synapses during sectioning.

\section{Number of appositions correlate with synaptic efficacy}

The amplitude of the postsynaptic response was correlated strongly with the number of putative synapses within each group of LACs, MACs, and WACs $(r=0.88,0.95$, and 0.99 , respectively). Strong correlations also were observed between the number of putative synapses and variance in EPSP amplitude within 


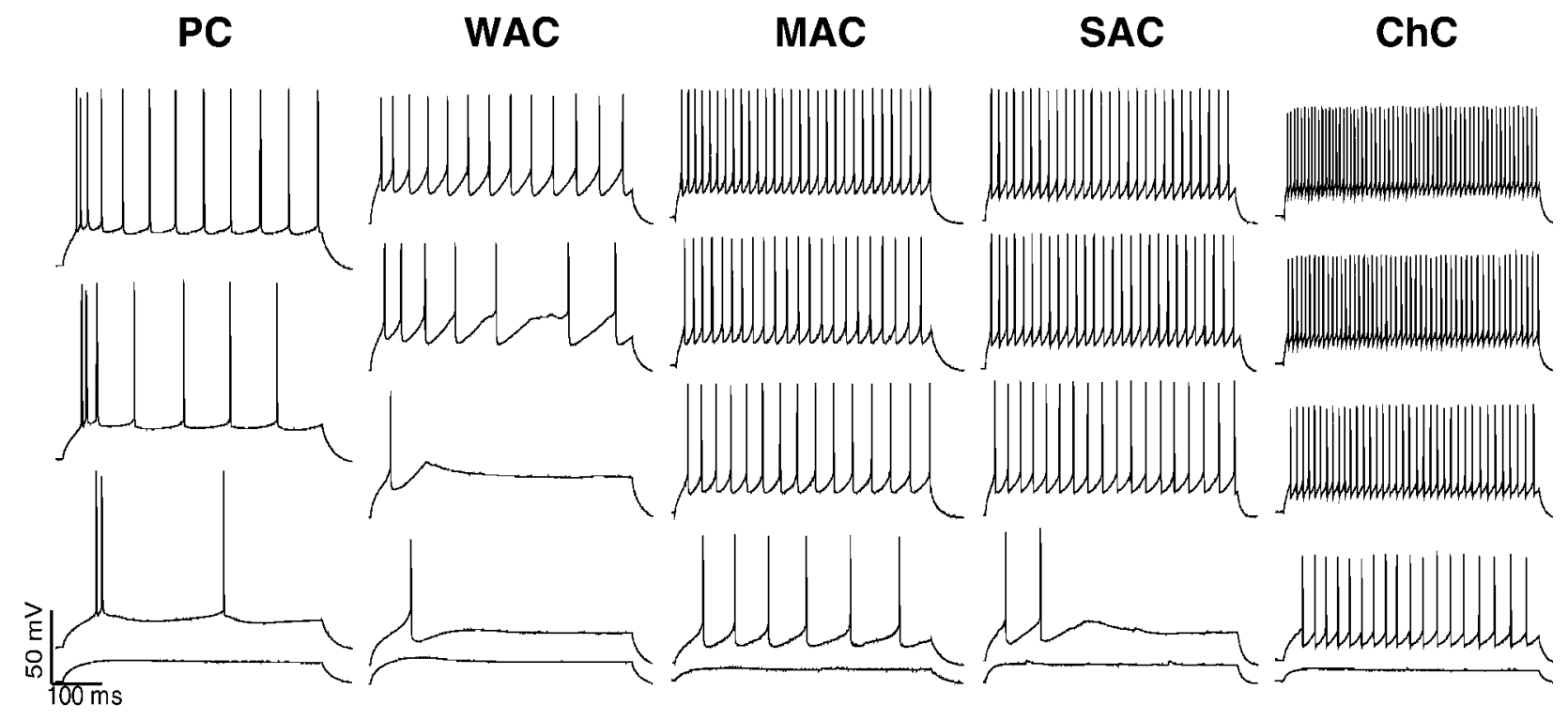

Figure 4. Distinctive membrane properties of the subtypes of postsynaptic interneurons. The consecutive traces (from the bottom to the top) are evoked neuronal responses by current pulse injections of $50 \mathrm{pA}$ increments and $500 \mathrm{msec}$ duration. The chandelier neuron $(C h C)$ displays the highest firing rates, with no spike adaptation. This is followed by the small arbor $(S A C)$ and then by medium arbor $(M A C)$ basket cells. Note that the MAC displays some spike adaptation. The wide arbor basket cell $(W A C)$ has low evoked firing rates and prominent spike adaptation. In fact, these two parameters are almost identical to those of pyramidal cells $(P C)$; hence the WAC does not really fall into the fast-spiking cell classification, as commonly defined. Nevertheless, it is distinguishable from pyramidal cells by its smaller and narrower action potentials, which are characteristic of interneurons.

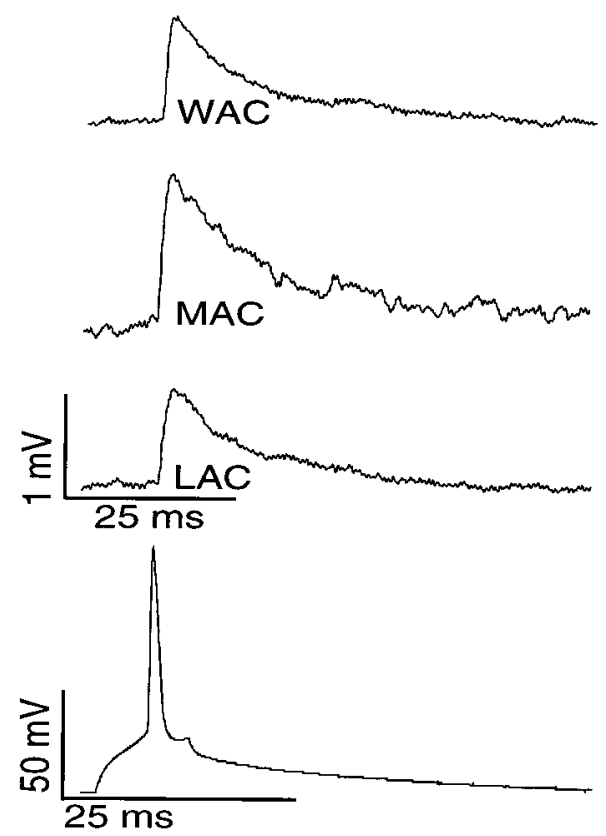

Figure 5. Unitary EPSPs recorded in local, medium, and wide arbor basket postsynaptic interneurons. Evoked action potential in presynaptic pyramidal cells (bottom trace) produced monosynaptic EPSPs in postsynaptic cells (top traces). One putative synapse in LAC (this pair is reconstructed in Fig. 2c) generated an average EPSP of $1 \mathrm{mV}$, as did three synapses in WAC (reconstructed in Fig. 3c). Two synapses in MAC (reconstructed in Fig. $3 a$ ) produced an average $1.5 \mathrm{mV}$ EPSP.

each of these groups ( $r=0.89,0.71$, and 0.99 , respectively). However, the same number of synapses produced a stronger response in the group of LACs than in MACs and especially in WACs. To calculate the postsynaptic depolarization produced by a single contact (unit EPSP), we divided the mean unitary EPSP by the number of putative synapses. We found a strong negative
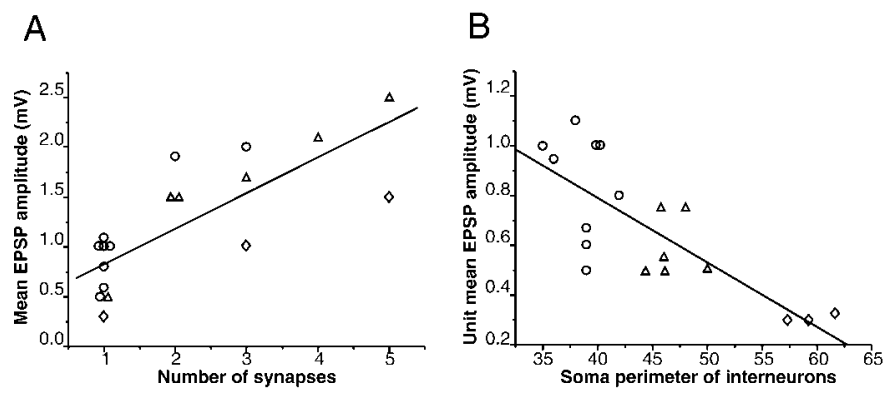

Figure 6. Structure-function correlates of intrinsic excitatory neurotransmission in the pyramidal-interneuron circuit. $A$, A strong positive correlation $(r=0.78)$ between the number of putative synapses and the strength of the postsynaptic response. $B$, A strong negative correlation $(r=-0.83)$ between the unit mean amplitude of EPSPs (calculated as the mean EPSP divided by the number of synapses) and the soma size of the postsynaptic interneurons, which correspond to distinct interneuronal subtypes. Circles, LACs; triangles, MACs; diamonds, WACs.

correlation $(r=-0.83 ; p<0.0001$; see also Fig. $6 B$ ) between the soma size of interneurons and the amplitude of unit EPSPs, indicating that a single synapse has a greater impact on LACs than on MACs and especially greater than on WACs. Indeed, one synapse usually produced the same $(\sim 1 \mathrm{mV})$ postsynaptic depolarization in LACs that three to five synapses did in the WACs (see Fig. 5A). This influence of postsynaptic interneuron obviously weakened across the correlation of the groups $(r=0.67$; Fig. $6 A$ ) as compared with within-groups correlation between the number of contacts and strength of response $(r=0.88,0.95$, and 0.99 for LACs, MACs, and WACs, respectively).

Interestingly, single connections across the groups had twofold smaller EPSP amplitudes ( 0.8 vs $1.6 \mathrm{mV}$, respectively; $p<0.0001$, one way ANOVA) and twofold lower variability (SD \pm 0.3 vs $\mathrm{SD} \pm 0.6 ; p<0.0001)$ as compared with multiple connections (Fig. 7). 


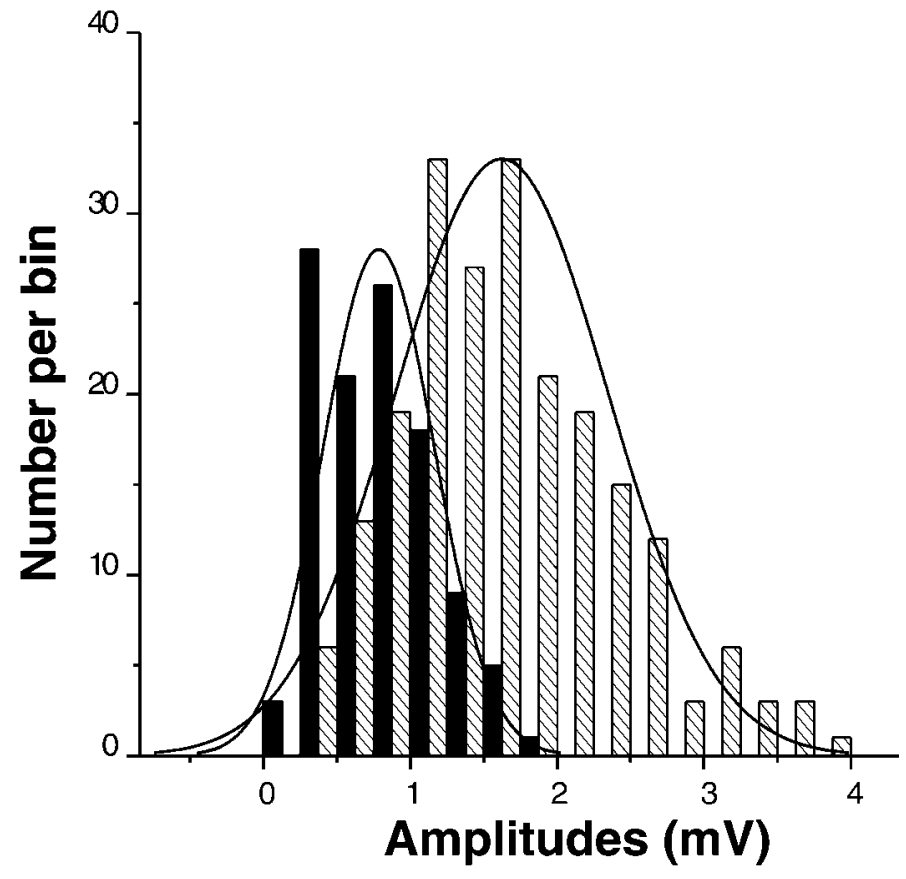

Figure 7. Distribution of the EPSP amplitudes in the identified pyramidal-to-interneuron connections. Both the amplitudes and variance for the group with single-contact connections ( filled columns) are twofold smaller than for the group of multi-contact connections (patterned columns).

\section{DISCUSSION}

\section{Anatomical and physiological properties of interneurons}

There is, as yet, no universally established classification scheme of neocortical interneurons. In the present study we have analyzed synaptic transmission between identified pyramidal neurons and three groups of interneurons in layers II/III of prefrontal cortex that are differentiated by the spread of their axonal arbors and, consequently, by the size of cortical territories and number of neurons each interneuron has the potential to innervate and inhibit. The width of axonal distribution of an interneuron also is correlated with the size of its soma. These groups of interneurons originally were described anatomically in the monkey prefrontal cortex, with LACs and WACS expressing parvalbumin immunoreactivity and MACs containing cholecystokinin (Lund and Lewis, 1993). In contrast, parvalbumin-containing interneurons in the rat have been categorized physiologically as a single group of fast-spiking cells. On the basis of these previous findings, we initially expected that local, medium, and wide arbor interneurons also would belong to one category of fast-spiking cells. However, the present findings demonstrate that each of these morphological groups has distinct physiological correlates that are based on their firing rates and spike widths. LACs (including $\mathrm{ChCs}$ ), MACs, and WACs, respectively, have progressively lower evoked firing rates and wider action potentials. Chandelier interneurons occupied one extreme of this order, demonstrating the highest evoked firing rates and the narrowest spikes, whereas WACs with the lowest firing rates and the widest action potentials were at the other extreme. Indeed, the low firing rates of WACs, often with considerable spike adaptation, resembled the firing pattern of pyramidal neurons, although they still could be differentiated from the latter by narrower spikes of lower amplitude.
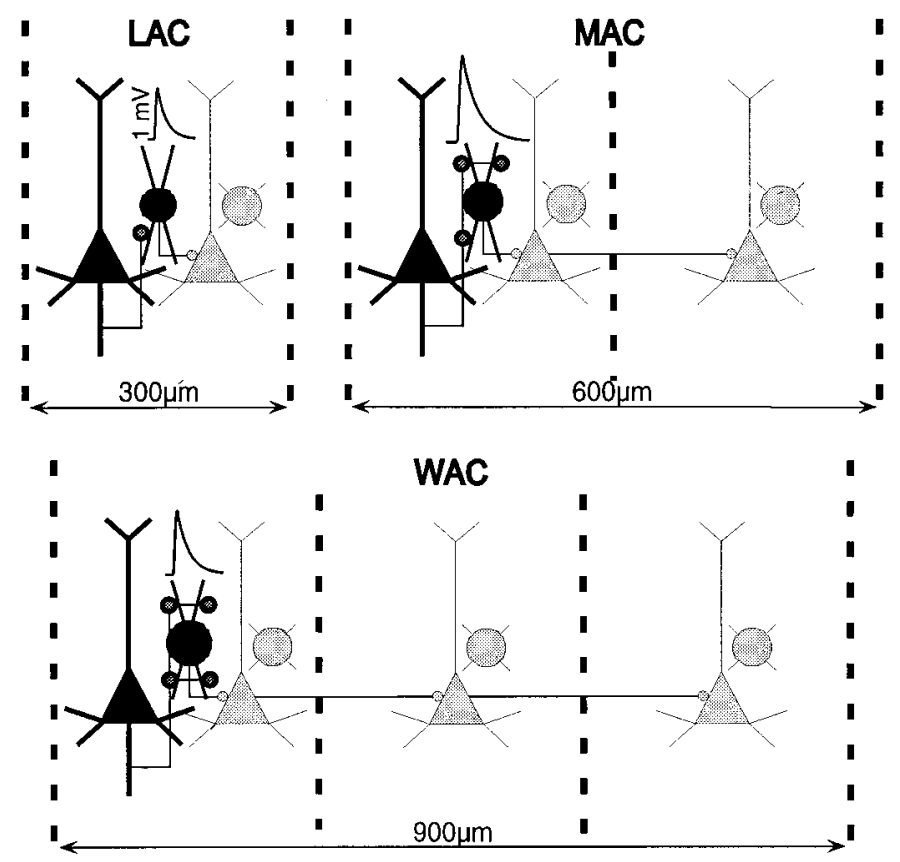

Figure 8. Summary diagram of the three types of interneurons and their associated EPSPs that were examined in the present study, illustrating the putative synaptic connections that were observed between pyramidal and interneuron pairs and the presumed targets of the local arbor $(L A C)$, medium arbor $(M A C)$, and wide arbor $(W A C)$ neurons (see Fig. 3 for documentation of the axonal arbors that are illustrated here).

Thus, it appears that fast-spiking interneurons can be differentiated further both physiologically and morphologically and, on this basis, can be predicted to have different roles in cortical function yet to be determined.

\section{Pyramidal cell innervation of LACs, MACs, and WACs}

Feedforward excitation from pyramidal neurons onto interneurons is a well established fact of cortical architecture (Shepherd, 1974), although there are more physiological investigations of feedforward inhibition onto principal neurons than the reverse (Eysel, 1992; Gulyas et al., 1993; Buhl et al., 1997; Eysel et al., 1998). However, the nature of inhibitory input on neurons in the same cortical column (presumably LACs) or in neighboring cortical columns (for MACs and WACs) is highly relevant to the function of lateral inhibitory mechanisms controlling neuronal excitability generally and receptive field conformation specifically. The present study is the first to examine pyramidal-interneuronal interactions in prefrontal cortex of any species and to correlate physiological properties within identified anatomical circuits. Here we demonstrate that interneurons with different widths of horizontal axonal spread and therefore with presumptive control over different cortical modules receive a distinctive excitatory unitary input from local pyramidal cells, among other possible sources of innervation. LACs were driven reliably by a single contact, similar to findings in hippocampal basket interneurons (Gulyas et al., 1993) but contrasting with very unreliable pyramidal-to-burst firing spiny interneuronal connections in rat somatomotor cortex (Deuchars and Thomson, 1995). The latter may be another example of an influence of postsynaptic interneurons on the properties of their synaptic input. More contacts (two to five) appear to be necessary for reliable neurotransmission in MACs and WACs because their efficacy, calculated per single 
contact, exhibited a progressive decline. One possible explanation for this decline may be a lower probability of neurotransmitter release in multisynaptic versus monosynaptic connections, as indicated by a twofold higher variance in EPSP amplitudes (SD \pm 0.66) with multiple as compared with single putative synapses (average SD \pm 0.33 ). Other possibilities include changes in membrane conductance and/or number and sensitivity of postsynaptic receptors. Clearly, further studies are necessary to address whether presynaptic or postsynaptic mechanisms are involved.

\section{Correlation of morphologically identified contacts, EPSP amplitudes, and type of postsynaptic interneuron}

The present study demonstrated that the number of synapses established by presynaptic pyramidal neurons on their postsynaptic targets correlated with the type of postsynaptic interneuron. Single synapse unitary connections generally appeared to be the rule for the group of LACs, because only one synapse was found in seven of nine pairs in the group. In contrast, two to five synapses per unitary connection usually were found in the two groups of MACs and WACs. The study also provides evidence of a strong structure-function correlation, that between the numbers of putative excitatory contacts on identified interneurons and synaptic efficacy. An important caveat in this study is that appositions between identified terminals and postsynaptic interneurons in this study were not confirmed at the electron microscopic (EM) level for several reasons. Although such analysis would be ideal, it is not feasible considering the fact that our light microscopic observation included 39 putative synaptic contacts. Despite that precaution, the following arguments make us confident in the numbers of estimated putative synapses. First, almost one-half ( 9 of 20) of the physiologically connected neuronal pairs had a single putative synapse, which strongly argues against light microscopic overestimation of contacts. Second, we have used an identical labeling protocol in a previous study and provided EM confirmation of our light microscopic analysis in the four of four contacts that were examined (Krimer et al., 1997). Also, other previous studies (Buhl et al., 1997; Markram et al., 1997) in which light electron microscopic correlation was performed similarly have found high (90-100\%) agreement between the light microscopic estimation and ultrastructural analysis of synaptic contacts. Third, our differential labeling of presynaptic and postsynaptic neurons by two chromogens not only enabled us to reconstruct neuronal pairs accurately but also allowed us to distinguish synapses reliably from autapses. Further, our anatomical estimation is supported strongly by the physiological data, which show a systematic, replicable, and highly significant association between putative synaptic number and synaptic efficacy in each subgroup of identified interneuron. Finally, the mean EPSP amplitude and mean variance for the multiple putative synapse connections were approximately twice as high as those for single putative synapse connections. This difference was highly significant.

Surprisingly, support in the literature for strong correlations between synaptic number and postsynaptic response generally has been sparse and weak. In a study similar to ours, Buhl et al. (1997) analyzed five pyramidal-to-interneuronal pairs in cat visual cortex. Three basket cells, one dendrite-targeting cell, and one double bouquet cell represented the postsynaptic interneurons. In these pairs the mean EPSPs of $0.3,0.4,1.2,0.9$, and $0.7 \mathrm{mV}$ corresponded to $1,2,2,4$, and 7 synapses, respectively. The limited number of observations precluded correlation analysis in that study. This issue also has been addressed in other model systems such as the goldfish Mauthner cell (Korn et al., 1986), layer IV stellate (Feldmeyer et al., 1999), and layer V tufted pyramids of rat somatosensory cortex (Markram et al., 1997), but strong correlations were not observed. In the goldfish Mauthner cell the number of identified synapses was quite extensive (3-52), yet only a trend correlation was observed and only among the neurons with small numbers of presynaptic terminals (Korn et al., 1986). It seems distinctly possible that multiple synaptic connections may be associated with a different probability of transmitter release, which could obscure any correlation between synaptic number and postsynaptic response. In support of this supposition, linear summation of mimicked EPSPs has been demonstrated in CA1 hippocampal pyramidal neurons when the presynaptic component was bypassed with local iontophoresis of glutamate (Cash and Yuste, 1999). The range of synapses examined in our study was only one to five, and this could favor the observed strong correlation between the number of appositions and EPSP amplitude. In addition, the different membrane properties of layer $\mathrm{V}$ pyramidal neurons (Markram et al., 1997), and stellate neurons of layer IV (Feldmeyer et al., 1999) as compared with the nonpyramidal neurons studied here also may account for the differences in the strength of correlations between these and our studies. For example, the amplitudes of both EPSPs (Stuart and Spruston, 1998) and backpropagated action potentials (Stuart and Sakmann, 1994; Spruston et al., 1995) are strongly attenuated in distal dendrites of pyramidal neurons. In contrast, at least in certain subtypes of interneurons these parameters are not affected significantly by dendritic location (Chitwood et al., 1999; Martina et al., 2000). In support of this, we did not observe any difference in response between putative synapses located distally or more proximally on the dendrites of interneurons.

\section{Implications for working memory circuitry}

The present study examined pyramidal-to-nonpyramidal interactions in prefrontal cortex for which the cortical modules in the primate are composed of neurons with "memory fields." In the primate visual memory system these neurons actively maintain mnemonic traces of the location of preferred spatial targets and often are inhibited in the direction opposite to the preferred target (Funahashi et al., 1989; Wilson et al., 1994; Rao et al., 1999). It has been proposed that an interneuron is a necessary intermediary between pyramidal neurons of opposite polarity, a proposition that entails input to an interneuron from a nearby pyramidal neuron and/or common input to both and an output of the interneuron to a distant pyramidal cell (Goldman-Rakic, 1995). The interneurons with differential horizontal axonal spans in the present study are candidates for these intermediary functions. LACs, MACs, or WACs all may share the memory field of an adjacent pyramidal cell by virtue of proximity, common input, and/or axon collaterals. However, the same subgroups are candidates for inhibiting pyramidal cells in progressively more remote columns for which the neurons have memory fields of adjacent, intermediate, and opponent polarities, respectively. Recent dualcell extracellular recordings in monkeys performing a spatial working memory task in this laboratory support this hypothesis: adjacent (within $50 \mu \mathrm{m}$ ) pyramidal-nonpyramidal pairs were found to share common specificities (within $60^{\circ}$ ) for the location of visual stimuli in the memory fields of the neurons (Constantinidis et al., 1999), whereas such pairs at distances $>200 \mu \mathrm{m}$ from each other had progressively greater disparities up to $120^{\circ}$ (Rao et al., 1999; Constantinidis et al., 2001). The present in vitro results add a synaptic dimension to this circuitry by revealing that 
the MACs and WACs are amplified both in number of pyramidal cell synapses and in their EPSP amplitude, possibly to compensate for the distance and size of their terminal fields, which presumably mediate cross-directional inhibition.

\section{REFERENCES}

Angulo MC, Staiger JF, Rossier J, Audinat E (1999) Developmental synaptic changes increase the range of integrative capabilities of an identified excitatory neocortical connection. J Neurosci 19:1566-1576.

Bugbee NM, Goldman-Rakic PS (1983) Columnar organization of corticocortical projections in squirrel and rhesus monkeys: similarity of column width in species differing in cortical volume. J Comp Neurol 220:355-364.

Buhl EH, Tamas G, Szilagyi T, Stricker C, Paulsen O, Somogyi P (1997) Effect, number, and location of synapses made by single pyramidal cells onto aspiny interneurons of cat visual cortex. J Physiol (Lond) 500:689-713

Cash S, Yuste R (1999) Linear summation of excitatory inputs by CA1 pyramidal neurons. Neuron 22:383-394.

Chen W, Zhang JJ, Hu GY, Wu CP (1996) Electrophysiological and morphological properties of pyramidal and nonpyramidal neurons in the cat motor cortex in vitro. Neuroscience 73:39-55.

Chitwood RA, Hubbard A, Jaffe DB (1999) Passive electrotonic properties of rat hippocampal CA3 interneurons. J Physiol (Lond) $515: 743-756$

Constantinidis C, Franowicz MN, Goldman-Rakic PS (1999) Multiple electrode analysis of local circuitry in the primate prefrontal cortex during spatial working memory. Soc Neurosci Abstr 25:44.1.

Constantinidis C, Franowicz MN, Goldman-Rakic PS (2001) Coding specificity in cortical microcircuits: a multiple electrode analysis of primate prefrontal cortex. J Neurosci 21:3646-3655.

Deuchars J, Thomson AM (1995) Innervation of burst firing spiny interneurons by pyramidal cells in deep layers of rat somatomotor cortex: paired intracellular recordings with biocytin filling. Neuroscience 69:739-755.

Elhanany E, White EL (1990) Intrinsic circuitry: synapses involving the local axon collaterals of corticocortical projection neurons in the mouse primary somatosensory cortex. J Comp Neurol 291:43-54.

Eysel UT (1992) Lateral inhibitory interactions in areas 17 and 18 of the cat visual cortex. Prog Brain Res 90:407-422.

Eysel UT, Shevelev IA, Lazareva NA, Sharaev GA (1998) Orientation tuning and receptive field structure in cat striate neurons during local blockade of intracortical inhibition. Neuroscience 84:25-36.

Feldmeyer D, Egger V, Lubke J, Sakmann B (1999) Reliable synaptic connections between pairs of excitatory layer 4 neurones within a single "barrel" of developing rat somatosensory cortex. J Physiol (Lond) 521:169-190.

Funahashi S, Bruce CJ, Goldman-Rakic PS (1989) Mnemonic coding of visual space in the monkey's dorsolateral prefrontal cortex. J Neurophysiol 61:331-349.

Galarreta M, Hestrin S (1998) Frequency-dependent synaptic depression and the balance of excitation and inhibition in the neocortex. Nat Neurosci 1:587-594.

Goldman-Rakic PS (1984) Modular organization of prefrontal cortex. Trends Neurosci 7:419-424.

Goldman-Rakic PS (1995) Cellular basis of working memory. Neuron 14:477-485.

Gulyas AI, Miles R, Sik A, Toth K, Tamamaki N, Freund TF (1993) Hippocampal pyramidal cells excite inhibitory neurons through a single release site. Nature 366:683-687.

Kawaguchi Y (1995) Physiological subgroups of nonpyramidal cells with specific morphological characteristics in layer II/III of rat frontal cortex. J Neurosci 15:2638-2655.

Kisvarday ZF, Kim DS, Eysel UT, Bonhoeffer T (1994) Relationship between lateral inhibitory connections and the topography of the orientation map in cat visual cortex. Eur J Neurosci 6:1619-1632.

Korn H, Faber DS, Triller A (1986) Probabilistic determination of synaptic strength. J Neurophysiol 55:402-421.

Krimer LS, Jakab RL, Goldman-Rakic PS (1997) Quantitative three- dimensional analysis of the catecholaminergic innervation of identified neurons in the macaque prefrontal cortex. J Neurosci 17:7450-7461.

Lund JS, Lewis DA (1993) Local circuit neurons of developing and mature macaque prefrontal cortex: Golgi and immunocytochemical characteristics. J Comp Neurol 328:282-312.

Markram H, Lubke J, Frotscher M, Roth A, Sakmann B (1997) Physiology and anatomy of synaptic connections between thick tufted pyramidal neurons in the developing rat neocortex. J Physiol (Lond) 500:409-440.

Martina M, Vida I, Jonas P (2000) Distal initiation and active propagation of action potentials in interneuron dendrites. Science 287:295-300.

McCormick DA, Connors BW, Lighthall JW, Prince DA (1985) Comparative electrophysiology of pyramidal and sparsely spiny stellate neurons of the neocortex. J Neurophysiol 54:782-806.

McGuire BA, Gilbert CD, Rivlin PK, Wiesel TN (1991) Targets of horizontal connections in macaque primary visual cortex. J Comp Neurol 305:370-392.

Melchitzky DS, Sesack SR, Pucak ML, Lewis DA (1998) Synaptic targets of pyramidal neurons providing intrinsic horizontal connections in monkey prefrontal cortex. J Comp Neurol 390:211-224.

Melchitzky DS, Gonzalez-Burgos G, Barrionuevo G, Lewis DA (2001) Synaptic targets of the intrinsic axon collaterals of supragranular pyramidal neurons in monkey prefrontal cortex. J Comp Neurol 430:209-221.

Muly 3rd EC, Szigeti K, Goldman-Rakic PS (1998) D1 receptor in interneurons of macaque prefrontal cortex: distribution and subcellular localization. J Neurosci 18:10553-10565.

Rao SG, Williams GV, Goldman-Rakic PS (1999) Isodirectional tuning of adjacent interneurons and pyramidal cells during working memory: evidence for microcolumnar organization in PFC. J Neurophysiol 81:1903-1916.

Rao SG, Williams GV, Goldman-Rakic PS (2000) Destruction and creation of spatial tuning by disinhibition: GABA blockade of prefrontal cortical neurons engaged by working memory. J Neurosci 20:485-494.

Reyes A, Lujan R, Rozov A, Burnashev N, Somogyi P, Sakmann B (1998) Target cell-specific facilitation and depression in neocortical circuits. Nat Neurosci 1:279-285.

Sanchez-Vives MV, McCormick DA (2000) Cellular and network mechanisms of rhythmic recurrent activity in neocortex. Nat Neurosci 10:1027-1034.

Shepherd G (1974) The synaptic organization of the brain: an introduction. New York: Oxford UP.

Sillito AM (1975) The contribution of inhibitory mechanisms to the receptive field properties of neurones in the striate cortex of the cat. J Physiol (Lond) 250:305-329.

Sillito AM (1984) Functional considerations of the operation of GABAergic inhibitory processes in the visual cortex. In: Cerebral cortex (Jones EG, Peters A, eds), pp 91-117. New York: Plenum.

Spruston N, Schiller Y, Stuart G, Sakmann B (1995) Activity-dependent action potential invasion and calcium influx into hippocampal CA1 dendrites. Science 268:297-300.

Stuart GJ, Sakmann B (1994) Active propagation of somatic action potentials into neocortical pyramidal cell dendrites. Nature 367:69-72.

Stuart GJ, Spruston N (1998) Determinants of voltage attenuation in neocortical pyramidal neuron dendrites. J Neurosci 18:3501-3510.

Stuart GJ, Dodt HU, Sakmann B (1993) Patch-clamp recordings from the soma and dendrites of neurons in brain slices using infrared video microscopy. Pfl $\int$ gers Arch 423:511-518.

Tarczy-Hornoch K, Martin KA, Jack JJ, Stratford KJ (1998) Synaptic interactions between smooth and spiny neurons in layer 4 of cat visual cortex in vitro. J Physiol (Lond) 508:351-363.

Thomson AM, West DC, Deuchars J (1995) Properties of single axon excitatory postsynaptic potentials elicited in spiny interneurons by action potentials in pyramidal neurons in slices of rat neocortex. Neuroscience 69:727-738.

Williams SM, Goldman-Rakic PS, Leranth C (1992) The synaptology of parvalbumin-immunoreactive neurons in the primate prefrontal cortex. J Comp Neurol 320:353-369.

Wilson FA, O'Scalaidhe SP, Goldman-Rakic PS (1994) Functional synergism between putative $\gamma$-aminobutyrate-containing neurons and pyramidal neurons in prefrontal cortex. Proc Natl Acad Sci USA 91:40094013. 